\title{
A rare case of ischemic colitis due to ergotamine
}

\begin{abstract}
Ergotamine toxicity is rare complication, including tachycardia, arterial spasm, or ischemic colitis which occurring as a result of a treatment with the drug du to different conditions. We report a 54-year-old woman using an ergot-derived drug for migraine. Diagnosis of ergotamine intoxication begins with suspicion. Every doctor and surgeons should be aware of this acute dangerous condition.
\end{abstract}

Keywords: Ischemic colitis; Ergotamine
Volume 5 Issue 5 - 2016

\author{
Funes C, Daneri, Cillo M, Ruiz H, Estefania D, \\ Patrón Uriburu JC \\ Department of Colorectal Surgery, British Hospital of Buenos \\ Aires, Argentina
}

\begin{abstract}
Correspondence: Juan Carlos Patron Uriburu, Department of Colorectal Surgery, British Hospital of Buenos Aires, Perdriel 74, Buenos Aires, Argentina, Email jcpatron@hotmail.com
\end{abstract}

Received: July 28, 2016 | Published: November 10, 2016

\section{Introduction}

Ischemic colitis is caused by the temporary deprivation of blood flow to the large bowel, due to a local or systemic alteration of the blood supply. Its incidence varies between 4,5 to 44 cases every 100,000 people per year. ${ }^{1}$ People affected by this entity are mainly female over 60 years old. ${ }^{2}$ However, there are cases reported in young people with no risk factors. Its presentation runs between mild transitory episodes to fulminant colitis that can lead to necrosis, perforation, peritonitis and death. The risk factors identified are high blood pressure, diabetes mellitus, dyslipidemia, coronary disease and drugs, such as NSAIDs, ergotamine, cocaine, antibiotics, cytotoxic drugs and vasoactive agents..$^{3-6}$

\section{Case report}

A 54-year-old female was admitted in the emergency department for 24 hour-longmigraines, diarrhea, lower gastrointestinal bleeding (15 episodes) and left lower abdominal pain. The patient is a former smoker (6 pack/year) with a record of dyslipidemia, thalassemia minor and chronic migraine treated with ergotamine suppositories.

Physical examination showed normal vital parameters, abdomen with mild defense and no peritoneal irritation. Traces of blood were found during the rectal examination.

i. Blood test results: $\mathrm{Hto}: 32 \% \mathrm{Hb}: 10.3 \mathrm{~g} / \mathrm{dl}$; WBC 16,100/mm3; KPTT 28 sec; Quick 99\%; Platelets 212,000/ $\mathrm{mm}^{3}$.

ii. Abdominal CT: Irregular wall thickening $(>3 \mathrm{~mm})$ along the left portion of the large bowel with no compromise of the rectum, pericolic fat stranding, submucosal edema and enhancement of the mucosa after intravenous contrast administration (Figure 1).

A complete colonoscopy with previous mechanical bowel preparation was performed. The findings include segmental erythema and areas with pale mucosa from the splenic flexure to the rectosigmoideal junction, with rectal spare. Petechial hemorrhages, haustrations edema and fibrin were also described. Biopsy specimens were obtained.

Histologic examination showed epithelium erosion, mucosal and sub mucosal hemorrhage, atrophic crypts and a decrease in the number of caliciform cells. These findings were consistent with ischemic colitis. Conservative treatment was established, with the suppression of the ergotamine suppositories. The patient was discharged 48 hours after admission. No recurrent symptoms were reported. A colonoscopy was performed 6 months after the diagnosis, which showed remission of endoscopic findings (Figure 2).

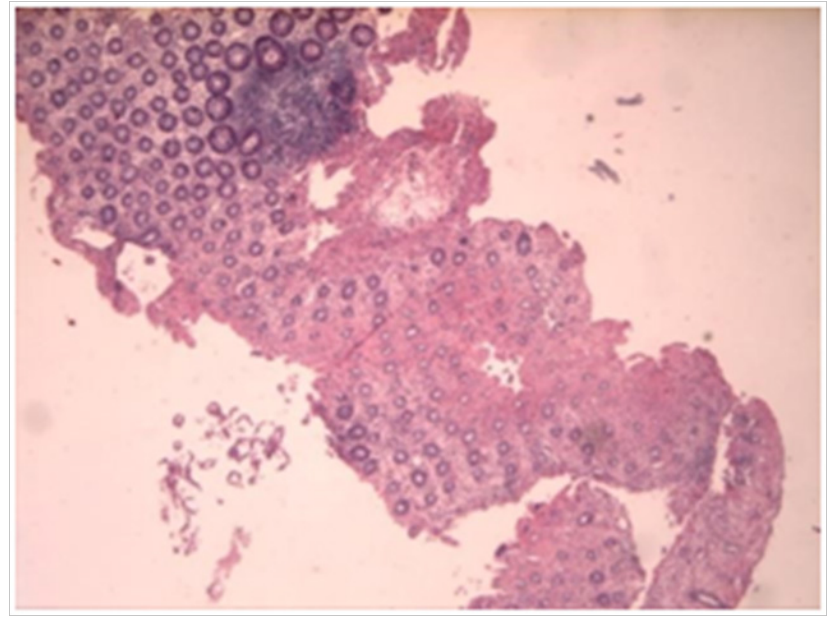

Figure I Histology.

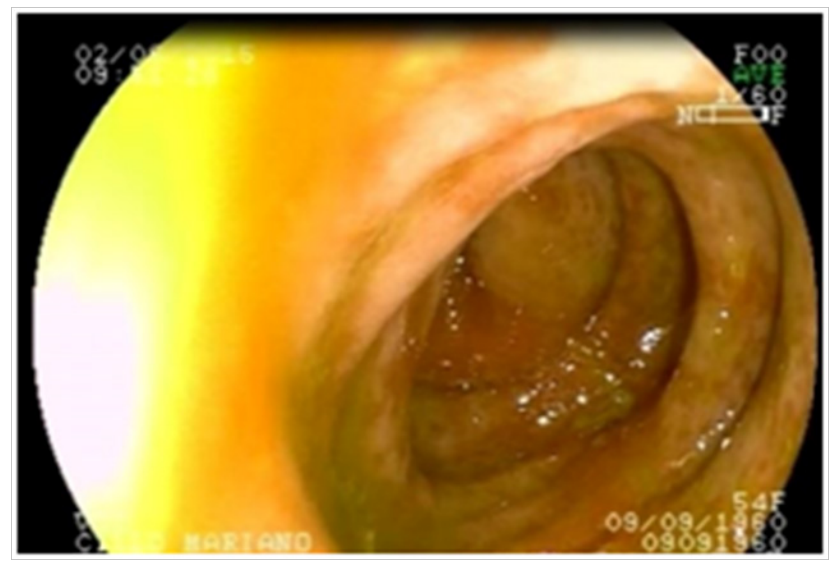

Figure 2 Colonoscopy:Ischemic colitis.

\section{Discussion}

Ischemic colitis is the most frequent form of intestinal ischemia and occurs when the large bowel is deprived of blood flow. A high level of suspicion is required for its diagnosis. Chronology of the symptoms (sudden onset abdominal pain, defecation urge and rectal bleeding) 
and clinical context should be considered. This data was validated in a multicentric prospective study in España CIE study, which showed that the suspicion rate for this entity in the emergency department was extremely low (24\%) 4 . There are two different clinical presentations:

1. Non gangrenous ischemic colitis $\mathbf{( 8 0 \% )}$ : Cramp like abdominal pain, localized on the left side, defecation urge and rectal bleeding or hematoquezia 12 to 24 hours from the beginning of the symptoms. ${ }^{7-9,10} 85 \%$ evolves with restitution ad integrum of the lesions, while $15 \%$ develop chronic ulcerative colitis.

2. Gangrenous ischemic colitis $(\mathbf{2 0} \%)$ : includes patients with transmural compromise of the large bowel that leads to necrosis and perforation. $10 \%$ of the patients have peritonitis at the moment of diagnosis. The mortality of this group ranges from 30 to $35 \% .3,4,11$ Unfortunately, there are no specific laboratory markers for early and accurate diagnosis.

CT Scan can provide useful information such as large bowel thickening, its extension, possible complications, and discharges differential diagnoses. ${ }^{12,13}$ Colonoscopy has high sensitivity and specificity to detect mucosal alterations and offers the possibility to take biopsy samples. ${ }^{8,11,14}$ It is highly recommended to perform it in the first 48 hours from the onset of symptoms, without previous mechanical bowel preparation, and avoiding excessive insufflation that could lead to even poorer blood flow.

The presence of the following findings suggests the existence of ischemic colitis:the spare of the rectum, segmental area of injury and hemorrhagic nodules that can also be detected in $\mathrm{x}$-ray scan (thumb printingsign. ${ }^{3,4,15,16}$ The treatment depends on the severity of the episode and its etiology. Most patients respond to conservative treatment with complete reversion of the mucosal damage. Surgical treatment should be limited to patients with severe complications ${ }^{17}$ (sepsis, peritonitis, stenosis, persistent or recurrent colitis).

The etiologic diagnosis of ischemic colitis includes:arterial occlusion, venous thrombosis and non-occlusive ischemia (systemic hypotension, inflammatory bowel disease, infectious colitis, NSAIDs induced colopathy, diverticular disease, vasoconstrictor drugs). ${ }^{10,18}$

Drug-induced ischemic colitis occurs after prolonged administration of suppositories with different chemicals and drugs, such as ergotamine. ${ }^{19}$ Ergotamine is a derivative rye ergot alkaloid, used in combination with acetaminophen and caffeine in the treatment of migraine. Rectal administration improves absorption and bioavailability to achieve the therapeutic effect. ${ }^{14,20}$

In this case report, the prolonged use of ergotamine suppositories, associated with the patient's comorbidities, lead us to the etiology of the patient's disease. CT scan, colonoscopy and pathological study of the biopsies confirmed the colitis origin. ${ }^{3,4,16}$ Ischemic colitis induced by ergotamine is fully resolved with the drug suspension, digestive rest, parenteral hydration and antibiotic therapy. ${ }^{20,21}$ Symptoms disappeared, and there was an integral restoration of the mucosa. ${ }^{4}$ There is a single report in which ergotamine induced ischemic colitis progressed to gangrene, requiring surgical treatment. ${ }^{22}$

In our case, conservative management was established with the suppression of suppositories, showing a complete clinical and endoscopic remission at follow-up. This is the sixth case of ischemic colitis due to ergotamine reported in the international literature. ${ }^{23}$

Diagnosis of ischemic colitis due to ergotamine begins with a high suspicion rate.It can then be established by comprehensive anamnesis, especially in patients with no vascular disease. Every physician working at an emergency department and surgeons should be aware of this infrequent condition. ${ }^{20,24}$

Drugs induced ischemic colitis is a rare entity, probably under diagnosed, and it should be considered ergotamine in ischemic colitis differential diagnosis.

\section{Acknowledgments}

None.

\section{Conflicts of Interset}

None.

\section{References}

1. Higgins PD, Davis KJ, Laine L. Systematic review:The epidemiology of ischemic colitis. Aliment Pharmacol Ther. 2004;19(7):729-738.

2. Green BT, Tendler DA. Ischemic colitis:A clinical review. South Med J. $2005 ; 98(2): 17-22$

3. BrandtLJ, Feuerstadt P, Blaszka MC. Anatomic patterns, patient characteristics and clinical outcomes in ischemic colitis:a study of 313 cases supported by histology. Am J Gastroenterol. 2010;105(10):2245-2252.

4. Montoro M, BrandtLJ, Santolaria S, et al. Clinical patterns and outcomes of ischemic colitis:Results of the Working Group for the Study of Ischemic Colitis in Spain (CIE study). Scand J Gastroenterol. 2011;46(2):236-246.

5. Longstreth GF, Yao JF. Epidemiology, Clinical Features, High-Risk Factors and Outcome of Acute Large Bowel Iischemia. Clinical Gastroenterol Hepatol. 2009;7(10):1075-1080.

6. Park CJ, Jang MK, Shin WG, et al. Can we predict the development of ischemic colitis among patients with lower abdominal pain. Dis Colon Rectum. 2007;50(2):232-238

7. BrandtLJ, Boley SJ. Colonic ischemia. Surg Clin North Am. 1992; 72:203-229.

8. Flobert C, Cellier C, Berger A, et al. Right colonic involvement is associated with severe forms of ischemic colitis and occurs frequently in patients with chronic renal failure requiring hemodialysis. $\mathrm{Am} J$ Gastroenterol. 2000;95(1):195-198.

9. Brandt LJ, Feuerstadt P. Intestinal Ischemia. In:Feldman M, Friedman L Brandt LJ (Eds), Gastrointestinal and Liver Disease. Pathophysiology, Diagnosis and Management. (9th edn), Saunders-Elsevier. Philadelphia, USA,2010;pp.2027-2048.

10. Feuerstadt P, Brandt LJ. Update on Colon Ischemia:Recent Insights and Advances. Curr Gastroenterol Rep. 2015;17(12):45.

11. Medina C, Vilaseca J, Videla S, et al. Outcome of patients with ischemic colitis:review of 53 cases. Dis Colon Rectum. 2004;47(2):180-184.

12. Sreenarasimhaiah J. Diagnosis and management of intestinal ischemic disorders. BMJ. 2003;326(7403):1372-1376.

13. Elder K, Lashner BA, Al Solaiman F. Clinical approach to colon ischemia. Cleve Clin J Med. 2009;76(7):401-409.

14. Bugallo F, Amarillo H. causa infrecuente de colitis isquémica. Revisión de la literatura. Reporte de caso. Rev ArgentColoproct. 2005.

15. Feuerstadt $P$, Brandt LJ. Colon Ischemia:Recent Insights and Advances. Curr Gastroenterol Rep. 2010;12(5):383-390.

16. Iacobellis F, Berritto D, Fleischmann D, et al. CT findings in acute, subacute, and chronic ischemic colitis:suggestions for diagnosis. BioMed Research International. 2014;2014:895248. 
17. Mosele M, Cardin F, Inelmen EM, et al. Ischemic colitis in the elderly:predictors of the disease and prognostic factors to negative outcome. Scand J Gastroenterol. 2010;45(4):428-433.

18. Sun D, Wang C, Yang L, et al. The predictors of the severity of ischemic colitis:a systematic review of 2823 patients from 22 studies. Colorectal Dis. 2016;18(10):949-958.

19. Tfelt-Hansen P, Saxena PR, Dahlof C, et al. Ergotamine in the acute treatment of migraine:A review and European consensus. Brain. 2000;123(pt 1):9-18.

20. Adam G, Kurt T, Cinar C, et al. Ergotamine-induced vasospastic ischemia mimicking arterial embolism:unusual case. Ulus Travma Acil Cerrahi Derg. 2014;20(4):291-294.
21. Mosli M, Parfitt J, Gregor J. Retrospective analysis of disease association and outcome in histologically confirmed ischemic colitis. J Dig Dis. 2013;14(5):238-243.

22. Nassif M, Ameer A, Meterissian SH, et al. A Case of unresectable rectal necrosis. Case Report Med. 2013;2011(2011):3.

23. Rodman RE, Willson TD, Connolly MM, et al. Ischemic Colitis Secondary to Ergotamine Use:A Case Study. Case Rep Gastroenterol. 2011;5(1):1-4.

24. Diaz Nieto R, Varcada M, Ogunbiyi OA, et al. Systematic review on the treatment of ischemic colitis. Colorectal Dis. 2011;13(7):744-747. 